\title{
Valproic acid inhibit non-small-cell lung cancer (A549 cell line) metastasis via inhibition of CD44v6 and Nm23H1
}

Professor Dr. Sorush Niknamian

Ph.D. in Cell and Molecular Biology, Board Member of Weston A Price Foundation, Washongton DC, United States

\begin{abstract}
Currently, epigenetic changes are considered to be one of the most important factors in the incidence of cancer and its metastasis to various tissues. It has been shown that CD44v6 and Nm23-H1 genes play a crucial role in the metastasis of various cancers. However, no study has been done on the effect of epigenetic factors on the expression of CD44v6 and Nm23-H1 genes in the lung cancer cell line, A549.Therefore, the present study investigated the role of a histone deacetylase inhibitor, valproic acid, on the expression of CD44v6 and Nm23-H1 genes and proteins in the A549 cell line. In this study, the A549 cell line was treated with valproic acid at concentrations of $2.2 \mathrm{mM}, 4.5 \mathrm{mM}$, and $9 \mathrm{mM}$. We then investigated the expression of CD44v6 and Nm23-H1 genes and proteins, as well as the expression of MMP-2 and MMP-9 genes and caspase-3 activity. The results showed that valproic acid significantly decreased the expression of CD44v6 gene and protein and MMP-2 and MMP-9 genes, but increased the expression of Nm23H1 gene and protein and the activity of caspase-3 $(\mathrm{p}<0.05)$. Our results showed that histone deacetylases affected the expression of CD44v6, Nm23-H1, MMP-2, and MMP-9, which are involved in metastasis. Therefore, the use of histone deacetylase inhibitors can be effective in the suppression of metastases and the treatment of lung cancer.
\end{abstract}

Key words: Valproic acid, Lung cancer, Metastases, Nm23H1, CD44v6 


\section{Background}

Lung carcinoma has a high metastatic capacity, rate of morbidity and mortality; it accounts for $17 \%$ of the total cancer cases in humans each year [1]. The predominant form of lung cancer is non-small cell lung cancer (NSCLC), which accounts for about 85\% of all lung cancer cases [2]. The ability of tumor cells to migrate to distant organs, which is called metastasis, is a frequent problem that accounts for about 30-40\% of NSCLC cases [3]. Multiple mechanisms and a variety of genetic interactions are involved in this process. Investigations into these mechanisms have led to the identification of stimulator, or proto-oncogene, and inhibitor, or tumor suppressor, genes. Therefore, one of the areas of research focusing on the control of metastases is the regulation of these genes.

Nm23-H1, which is also known as NDPK-A or NME1, is a metastatic suppressor gene in a variety of cancers [4]. Because of the various biological functions of Nm23-H1, its expression was downregulated in highly metastatic tumors. One study reported that mice lacking the Nm23-M1 gene developed lung cancer more frequently than the wild-type controls [5]. Various mechanisms have been reported to involve Nm23-H1 antitumor activities through the down-regulation of multiple growth factors and the regulation of multiple matrix metalloproteinases (MMPs) [6].

However, the expression of $\mathrm{Nm} 23-\mathrm{H} 1$ is highly regulated by genetic and epigenetic factors, such as histone deacetylases (HDAC). Valproic acid (VPA) is a confirmed HDAC inhibitor that belongs to a promising new class of antineoplastic agents. The positive effects that the administration of VPA has on Nm23-H1 and on the attenuation of breast cancer cell proliferation have been reported [7]. The migration of cells during the metastatic process involves adhesion molecules, such as transmembrane glycoproteins [8]. CD44 is a multifunctional transmembrane glycoprotein that has a role in cell-cell and cell-matrix interactions during tumorigenesis, angiogenesis, tumor growth, and metastasis [9]. Particular attention has been given to CD44v6 in cell-cell and cell-matrix interactions in tumorigenesis [10]. 
Studies have proven the importance of this protein in adhering to the base membranes and increasing the motility of cancer cells [11]. Recently, the importance of the inhibition of CD44v6 concomitant with matrix metalloproteinase-9 (MMP-9) has been reported [12].

Accordingly, the present investigation focused on the mechanisms of HDAC inhibition to amplify Nm23-H1 expression and halt CD44v6 expression; we also examined cell migration and metastasis of the NSCLC cell line A549.

\section{Materials and methods}

\section{Ethics Statement}

The study uses existing cultured cell lines only, and also the Baqiyatallah University of Medical Sciences's Ethics Committee also approved the procedures that were used in this study (code: IR. BMSU.REC.1395.1203).

\section{Cell Culture}

The human NSCLC line A549 was grown in Dulbecco's modified Eagle's medium (DMEM /F-12 with GlutaMAX, Gibco, 10565018; Thermo Fisher Scientific Inc., Waltham, MA), which was supplemented with $10 \%$ fetal bovine serum (Gemini Bio Products, West Sacramento, CA), 100 $\mathrm{U} / \mathrm{mL}$ penicillin $\mathrm{G}$, and $10 \mathrm{mg} / \mathrm{mL}$ streptomycin (Invitrogen, Carlsbad, CA), in disposable plastic bottles at $37^{\circ} \mathrm{C}$ in a humidified atmosphere of $5 \% \mathrm{CO}_{2}$. The culture medium was refreshed every 3-4 days using $0.25 \mathrm{mg} / \mathrm{mL}$ trypsin/EDTA (Invitrogen).

\section{MTT assay}

The viability of the A549 cells treated with VPA was assessed using the standard 3-(4, 5dimethylthiazol-2-yl)-2, 5-diphenyltetrazolium bromide (MTT) assay. Briefly, the cells were seeded at $10^{4} /$ cells per well in a 96-well plate and incubated overnight at $37^{\circ} \mathrm{C}$ with $5 \% \mathrm{CO}_{2}$. The medium was discarded and replaced with fresh medium the next day, and the cells were treated with various concentrations (0-16 mM) of VPA. After $24 \mathrm{~h}, 48 \mathrm{~h}$, and $72 \mathrm{~h}$, the MTT solution was added to a final concentration of $500 \mu \mathrm{g} / \mathrm{mL}$ and the plate was incubated at $37^{\circ} \mathrm{C}$ for $4 \mathrm{~h}$ in the dark. Finally, the supernatant was discarded and $150 \mu \mathrm{L}$ of DMSO was added to each well. The absorbance was measured at $570 \mathrm{~nm}$ with a reference filter of $630 \mathrm{~nm}$ using the Synergy H1 Hybrid Multi-Mode Reader (BioTek Instruments, Inc., Winooski, VT). The percentage of treated cells was compared with the percentage of control cells growing in the absence of VPA on a similar culture plate [13]. The IC50 was determined using GraphPad Prism software version 7.03 . 


\section{Measurement of caspase-3 activity}

To measure the caspase-3 activity, a caspase-3 substrate (DEVD-pNA, BioVision, Inc., Milpitas, CA) was reacted with a cell lysate substrate and the absorbance of the released pNA at $405 \mathrm{~nm}$ was measured. For this purpose, cells that were treated with multiple doses of VPA were lysed with chilled cell lysis buffer (BioVision, Inc.). After measuring the protein concentration of cell lysate using the bicinchoninic acid (BCA) method, equal volumes of protein $(100 \mu \mathrm{g})$ were diluted to a total volume of $50 \mu \mathrm{L}$ and mixed with $50 \mu \mathrm{L}$ of $2 \mathrm{X}$ reaction buffer (BioVision, Inc.). The caspase- 3 substrate was added and the mixture was incubated at $37^{\circ} \mathrm{C}$ for $2 \mathrm{~h}$ [14]. Finally, the absorbance was measured at $405 \mathrm{~nm}$ and the various groups were compared.

\section{RNA extraction and real-time polymerase chain reaction (RT-PCR)}

The total RNA was extracted using RNX Plus reagent (Cinnagen, Iran) according to the manufacturer's guideline and based on the phenol-guanidinium thiocyanate technique. Absorbance ratios of 260/230 and 260/280 were used with a NanoDrop spectrophotometer (BioTek, USA) to evaluate the quality and quantity of extracted RNA. The method was followed by DNase I digestion (Thermo Fisher Scientific Inc.). Complementary DNA (cDNA) was created using the PrimeScript RT reagent kit (Thermo Fisher Scientific Inc.). Real-time polymerase chain reaction (RT-PCR) was performed using SYBR Green Master Mix (Parstoos, Iran) and ABI Step One Real-Time PCR System (Applied Biosystems, Foster City, CA). The list of primers for the specific genes are shown in Table 1. All tests were done at least three times under the following conditions: $10 \mathrm{~min}$ at $95^{\circ} \mathrm{C}, 15 \mathrm{~s}$ for $95^{\circ} \mathrm{C}$, and $30 \mathrm{~s}$ at $60^{\circ} \mathrm{C}$. The melting curve analysis was performed from $60^{\circ} \mathrm{C}$ to $95^{\circ} \mathrm{C}$. The relative gene expression was calculated as $2^{-\Delta \Delta \mathrm{Ct}}[15]$.

Table 1 : Primer Sequences Were Used in RT-PCR.

\begin{tabular}{|c|l|l|}
\hline Gene & F/R & Primer sequences (5'-3') \\
\hline & & \\
\hline \multirow{2}{*}{ Nm23H1 } & Forward & TTAATCAGATGGTCGGGGAT \\
\cline { 2 - 3 } & Reverse & GATCTATGAATGACAGGAGG \\
\hline \multirow{2}{*}{ CD44V6 } & Forward & GTCGATGCTAGCTAGCCGTAGCATG \\
\cline { 2 - 3 } & Reverse & CGAGCTAGTCGTAGTCGATCGATCG \\
\hline \multirow{2}{*}{ MMP2 } & Forward & TCTCCTGACATTGACCTTGGC \\
\cline { 2 - 3 } & Reverse & CAAGGTGCTGGCTGAGTAGATC \\
\hline \multirow{2}{*}{ MMP9 } & Forward & CCTTGTGCTCTTCCCTGGAG \\
\cline { 2 - 3 } & Reverse & GGCCCCAGAGATTTCGACTC \\
\hline \multirow{2}{*}{ GAPDH } & Forward & AATCCCATCACCATCTTCCA \\
\cline { 2 - 3 } & Reverse & TGGACTCCACGACGTACTCA \\
\hline
\end{tabular}




\section{Western blotting}

The protein extraction and blotting procedure was performed on the cells seeded in a 6-well plate. The cells were scratched and transferred to a microtube by suspending them in RIPA lysis buffer (Santa Cruz Biotechnology, Inc., Dallas, TX), which contained protease inhibitors (SigmaAldrich, St. Louis, MO). After the cells were disrupted by sonication and centrifugation for $10 \mathrm{~min}$ at $14,000 \mathrm{rpm}$ and $4^{\circ} \mathrm{C}$, the protein content of the supernatant was evaluated using the BCA method. For each group, a total of $50 \mu \mathrm{g}$ protein was resolved and separated using SDS-PAGE on $10 \%$ polyacrylamide gels. They were then transferred to nitrocellulose membranes (Millipore) using a semi-dry transfer membrane system (Cleaver Scientific Ltd, Warwickshire, United Kingdom). After blocking the membrane for $1 \mathrm{~h}$ with 5\% skim milk in TBS buffer containing 20 $\mathrm{mM}$ Tris- $\mathrm{HCl}$ and $500 \mathrm{mM} \mathrm{NaCl}(\mathrm{pH}=7.4)$, the membrane was incubated with the indicated antibodies, mouse anti-human NM23-H1 antibody (Santa Cruz Biotechnology, Inc.) and mouse anti-human CD44v6 antibody (Abcam, Cambridge, UK), which were diluted in TBST buffer containing $20 \mathrm{mM}$ Tris- $\mathrm{HCl}, 500 \mathrm{mM} \mathrm{NaCl}$, and $0.5 \%$ Tween $20(\mathrm{pH}=7.4 ; 1: 1000)$ overnight at $4^{\circ} \mathrm{C}$. Then it was incubated for $1 \mathrm{~h}$ at $37^{\circ} \mathrm{C}$ with a secondary HRP-conjugated anti-mouse antibody (Santa Cruz Biotechnology, Inc.) that was diluted in TBST buffer (1:10000). Finally, enhanced chemiluminescence (Thermo Fisher Scientific Inc.) was used followed by exposure to radiographic film to detect secondary antibody binding.

\section{Statistical Analysis}

All tests were done in triplicate and statistical comparisons between groups were performed with one-way ANOVA followed by post hoc Tukey test. Data were expressed as mean \pm SD and $p<0.05$ considered as significant value.

\section{Results}

\section{Cytotoxicity of VPA in A549 Cells}

Cells expose to AVP at different concentration $(0-16 \mathrm{mM})$ and over different incubation periods of 24, 48 and $72 \mathrm{~h}$. The MTT results shows that AVP inhibits growth A549 cells in a concentration- 
and time- dependent manner, Fig 1. The IC50 values of AVP for A549 cells were 10.5, 6.8 and $4.5 \mathrm{mM}$ for 24,48 and $72 \mathrm{~h}$ respectively.

\section{VPA promotes caspase-3 activity in A549 cells}

An early marker of cell apoptosis, caspase-3 activity, was also determined to confirm the apoptotic effect of VPA in A549 cells cell line. Accordingly, the significant higher caspase-3 activity was observed by $9 \mathrm{mM}$ concentration of VPA for $72 \mathrm{~h}$ compare with other concentrations $(P<0.001)$. The results of caspase- 3 activity are shown in Fig. 2.

\section{VPA suppresses (MMP-2 and MMP-9) gene expression}

To investigate the suppressive effect of VPA against cell migration, A549 cells were examined to see if the ability of cell migration was affected by this agent. As it is shown in (fig. 3A and B), the doses with the highest cell apoptotic and cytotoxic effects could also hinder MMP-2 and MMP-9 gene expression and therefore cell migration through it. Such that, significant lower gene expression of MMP-2 and MMP-9 was observed in comparison with other doses $(P<0.01$ and $P<0.001)$.

\section{VPA promotes Nm23H-1 and alleviate CD44v6 expression in A549 cells}

To evaluate the mechanism of the inhibitory effect of VPA, changes in expression of Nm23-H1 and CD44v6 genes and proteins were investigated in A549 cells treated with multiple dose of VPA for $72 \mathrm{~h}$. The high up-regulation of this gene was also disclosed in cells treated with $9 \mathrm{mM}$ VPA $(P<0.0001)$. Similarly, higher protein expression of Nm23-H1 was also demonstrated in $9 \mathrm{mM}$ treatment. Gene expression analysis of CD44v6 in the cells treated with VPA for $72 \mathrm{~h}$ was also performed to compare it with no treated cells. The gene expression result suggests that VPA treatment significantly alleviate CD44v6, especially in higher dose $(P<0.0001)$. Interestingly, concentration related lower protein expression was also seen in a western blot examination, which in line with gene expression. The results of the expression of Nm23-H1 and CD44v6 genes and proteins are shown in (Fig 4A and B) \& (Fig. 5A and B and C) respectively.

\section{Discussion}

In this study, we focused on using an epigenetic mechanism to alter cellular behavior. Effective compounds were used on one of the most important epigenetic mechanisms, acetylation. We investigated the effects of VPA on the viability of human cell lung cancer A549 for 72 h.However, there was a decrease in the viability of cells as the concentration of VPA increased. There was also a significant higher gene and protein expression of $\mathrm{Nm} 23-\mathrm{H} 1$ and a lower gene and protein 
expression of CD44v6 in groups treated with VPA. Interestingly, the inhibitory effect of VPA involved inducing apoptosis and increasing caspase-3 activity.

$\mathrm{Nm} 23-\mathrm{H} 1$ is a protein with nucleoside diphosphate kinase activity, histidine kinase activity, and DNase activity[16]. The present investigation confirmed that the up-regulation of Nm23-H1 can induce DNA damage and subsequently increase genomic instability [17] Based on the inhibitory effect of VPA on HDAC, it seems that the stimulation of the Nm23-H1 gene is related to the same mechanism. Scientific evidence supports the key role that HDAC plays in down-regulating a set of metastasis promoter genes, which repress the effects of cancer cell invasion and metastasis in vitro and in vivo $[18,19]$.

Studies have shown the antineoplastic activity of HDACs in hematologic and solid malignancies The effects of HDAC inhibitors on tumorigenesis include halting migration, invasion, and growth and inducing apoptosis $[20,21]$. In the present investigation, VPA was seen to have a similar effect in the induction of apoptosis by prompting Nm23-H1. In another study, VPA increased the acetylation effect of histone and tubulin, which induced apoptosis in a gastric cell line [22]. The apoptotic effect of VPA on the lung cancer cell line A549 was confirmed in the present investigation by an increase in caspase- 3 activity. This result agreed with a previous study that reported the up-regulatory effect of VPA on caspase-3 expression [23].

CD44 is a cell-surface glycoprotein that plays a role in mediating mechanisms that involve cellcell and cell-matrix adhesion, such as blood cell generation, cell migration, and metastasis. Among all isoforms, CD44v6 contains a mutation of exon 11 and plays an important role in enhancing the adhesive ability of tumor cells [24]. An evidence-based report showed that an increase in the expression of CD44v6 altered the physiochemical properties of tumor cells and increased their metastatic potential [25]. The present study showed that the expression of CD44v6 protein and mRNA were concentration dependent on VPA, which demonstrated the inhibitory effect of VPA on tumorigenesis.

The adhesive process between cancer cells and the basal epidermal layer, which includes collagen, integrin, and fibronectin, requires mediation by CD44v6. This mediator creates the proper conditions for cancer cells to degrade the extracellular matrix (ECM) and for type I collagenases, such as MMP-2 and MMP-9, to invade [26]. MMP-2 and MMP-9 are zinc-dependent degrading enzymes that are involved in the degradation of the ECM and, thus, play a role in the metastasis 
activity of tumor cells [27]. The down-regulation of the MMP-2 and MMP-9 gene expression was observed in the present investigation.

In the present investigation, the up-regulation of $\mathrm{Nm} 23-\mathrm{H} 1$ was seen concomitant with the downregulation of MMP-9, which affected the metastatic capability. However, the relationship between Nm23-H1 and MMP-9 is controversial. For example, one study reported that Nm23-H1 caused an increase in MMP-9 gene expression and its gelatinolytic activity [28]. However, Nm23-H1 has caused suppressive effects without modifying the MMP-9 expression [29]. Moreover, the inhibition of the MMP-9 gene was also reported in relation to an increase in Nm23-H1 expression [30]. In the present investigation, an increased $\mathrm{Nm} 23-\mathrm{H} 1$ expression was seen with a decrease in expression of CD44v6, MMP-2, and MMP-9, which resulted in an increase in human cell lung cancer A549 apoptosis and caspase-3 activity.

In conclusion, our investigation demonstrated the antitumor activity of VPA against NSCLC. One possible mechanism was through the inhibition of HDAC, which resulted in an increase in Nm23H1 expression. This mechanism inhibited the process of cellular adhesion and matrix degradation by controlling the expression of CD44v6, MMP-2, and MMP-9, which affected the metastasis ability. This result could be helpful in future studies that investigate the mechanism-based inhibitory effect of VPA against cancer cells.

\section{Funding}

This experiment was kindly supported by Human Genetics Research Center, Baqiyatallah University of Medical Sciences, Tehran, Iran (Grant No.: 95-12-001243).

\section{Disclosure Statement}

No competing financial interests exist.

\section{References}

1. Jemal A, Bray F, Center MM, Ferlay J, Ward E, Forman D. Global cancer statistics. CA Cancer J Clin 2011,61:69-90.

2. Carbone DP, Gandara DR, Antonia SJ, Zielinski C, Paz-Ares L. Non-Small-Cell Lung Cancer: Role of the Immune System and Potential for Immunotherapy. J Thorac Oncol 2015,10:974-984.

3. Little AG, Gay EG, Gaspar LE, Stewart AK. National survey of non-small cell lung cancer in the United States: epidemiology, pathology and patterns of care. Lung Cancer 2007,57:253-260.

4. Tong Y, Yung LY, Wong YH. Metastasis suppressors Nm23H1 and Nm23H2 differentially regulate neoplastic transformation and tumorigenesis. Cancer Lett 2015,361:207-217. 
5. Boissan M, Wendum D, Arnaud-Dabernat S, Munier A, Debray M, Lascu I, et al. Increased lung metastasis in transgenic NM23-Null/SV40 mice with hepatocellular carcinoma. J Natl Cancer Inst 2005,97:836-845.

6. Huang C-S, Liao J-W, Hu M-L. Lycopene inhibits experimental metastasis of human hepatoma SK-Hep-1 cells in athymic nude mice. The Journal of nutrition 2008,138:538543.

7. Li GF, Qian TL, Li GS, Yang CX, Qin M, Huang J, et al. Sodium valproate inhibits MDA-MB231 breast cancer cell migration by upregulating NM23H1 expression. Genet Mol Res 2012,11:77-86.

8. Hanahan D, Weinberg RA. Hallmarks of cancer: the next generation. Cell 2011,144:646674.

9. Gunthert U, Hofmann M, Rudy W, Reber S, Zoller M, Haussmann I, et al. A new variant of glycoprotein CD44 confers metastatic potential to rat carcinoma cells. Cell 1991,65:13-24.

10. Herrera-Gayol A, Jothy S. Adhesion proteins in the biology of breast cancer: contribution of CD44. Exp Mol Pathol 1999,66:149-156.

11. Marhaba R, Zoller M. CD44 in cancer progression: adhesion, migration and growth regulation. J Mol Histol 2004,35:211-231.

12. Wei L, Yao Y, Zhao K, Huang Y, Zhou Y, Zhao L, et al. Oroxylin A inhibits invasion and migration through suppressing ERK/GSK-3beta signaling in snail-expressing non-small-cell lung cancer cells. Mol Carcinog 2016,55:2121-2134.

13. Kalantar H, Sabetkasaei M, Shahriari A, Hoseini MHM, Mansouri S, Kalantar M, et al. The Effect of Rapamycin on Oxidative Stress in MCF-7 and MDA MB-231 Human Breast Cancer Cell Lines. Jundishapur Journal of Natural Pharmaceutical Products 2016,11.

14. Liao H-F, Pan C-H, Chou P-Y, Chen Y-F, Wu T-S, Sheu M-J, et al. Toxicological effects of NCKU-21, a phenanthrene derivative, on cell growth and migration of A549 and CL1-5 human lung adenocarcinoma cells. PLoS One 2017,12:e0185021.

15. Mollaei H, Safaralizadeh R, Babaei E, Abedini MR, Hoshyar R. The anti-proliferative and apoptotic effects of crocin on chemosensitive and chemoresistant cervical cancer cells. Biomedicine \& Pharmacotherapy 2017,94:307-316.

16. Pandey S, Robertson ES. Oncogenic Epstein-Barr virus recruits Nm23-H1 to regulate chromatin modifiers. Lab Invest 2017.

17. Li Y, Nie CJ, Hu L, Qin Y, Liu HB, Zeng TT, et al. Characterization of a novel mechanism of genomic instability involving the SEI1/SET/NM23H1 pathway in esophageal cancers. Cancer Res 2010,70:5695-5705.

18. Shen WT, Wong TS, Chung WY, Wong MG, Kebebew E, Duh QY, et al. Valproic acid inhibits growth, induces apoptosis, and modulates apoptosis-regulatory and differentiation gene expression in human thyroid cancer cells. Surgery 2005,138:979-984; discussion 984-975.

19. D'Souza A, Onem E, Patel P, La Gamma EF, Nankova BB. Valproic acid regulates catecholaminergic pathways by concentration-dependent threshold effects on TH mRNA synthesis and degradation. Brain Res 2009,1247:1-10.

20. Lagneaux L, Gillet N, Stamatopoulos B, Delforge A, Dejeneffe M, Massy M, et al. Valproic acid induces apoptosis in chronic lymphocytic leukemia cells through activation of the death receptor pathway and potentiates TRAIL response. Exp Hematol 2007,35:15271537. 
21. Fortunati N, Bertino S, Costantino L, Bosco O, Vercellinatto I, Catalano MG, et al. Valproic acid is a selective antiproliferative agent in estrogen-sensitive breast cancer cells. Cancer Lett 2008,259:156-164.

22. Yagi Y, Fushida S, Harada S, Kinoshita J, Makino I, Oyama K, et al. Effects of valproic acid on the cell cycle and apoptosis through acetylation of histone and tubulin in a scirrhous gastric cancer cell line. J Exp Clin Cancer Res 2010,29:149.

23. Liu X, Chen L, Sun F, Zhang G. Enhanced suppression of proliferation and migration in highly-metastatic lung cancer cells by combination of valproic acid and coumarin-3carboxylic acid and its molecular mechanisms of action. Cytotechnology 2013,65:597-608.

24. Avoranta ST, Korkeila EA, Syrjänen KJ, Pyrhönen SO, Sundström JTT. Lack of CD44 variant 6 expression in rectal cancer invasive front associates with early recurrence. World Journal of Gastroenterology : WJG 2012,18:4549-4556.

25. Sun W, Chen G. Impact and mechanism of non-steroidal anti-inflammatory drugs combined with chemotherapeutic drugs on human lung cancer-nude mouse transplanted tumors. Oncology Letters 2016,11:4193-4199.

26. Protin U, Schweighoffer T, Jochum W, Hilberg F. CD44-deficient mice develop normally with changes in subpopulations and recirculation of lymphocyte subsets. J Immunol 1999,163:4917-4923.

27. Maehara Y, Kabashima A, Koga T, Tokunaga E, Takeuchi H, Kakeji Y, et al. Vascular invasion and potential for tumor angiogenesis and metastasis in gastric carcinoma. Surgery 2000,128:408-416.

28. Kuppers DA, Lan K, Knight JS, Robertson ES. Regulation of Matrix Metalloproteinase 9 Expression by Epstein-Barr Virus Nuclear Antigen 3C and the Suppressor of Metastasis Nm23-H1. Journal of Virology 2005,79:9714-9724.

29. Khan $\mathrm{MH}$, Yasuda $\mathrm{M}$, Higashino $\mathrm{F}$, Haque $\mathrm{S}$, Kohgo $\mathrm{T}$, Nakamura $\mathrm{M}$, et al. nm23-H1 suppresses invasion of oral squamous cell carcinoma-derived cell lines without modifying matrix metalloproteinase-2 and matrix metalloproteinase-9 expression. Am J Pathol 2001,158:1785-1791.

30. Ji $Q$, Zheng GY, Xia W, Chen JY, Meng XY, Zhang $H$, et al. Inhibition of invasion and metastasis of human liver cancer HCCLM3 cells by portulacerebroside A. Pharm Biol 2015,53:773-780. 


\section{Figure legend:}

Fig. 1. The effect of VPA $(0-16 \mathrm{Mm})$ on the cell viability of A549 cells after 24, 48 and $72 \mathrm{~h}$ incubation. Results are expressed as means $\pm \mathrm{S} \mathrm{EM}, \mathrm{n}=3$.

Fig. 2. Relative caspase 3 activity was determined in 549 cell line treated with $2.2,4.5$ and $9 \mathrm{mM}$ of VPA for 72 h. * $(P<0.01), * *(P<0.001)$ compared to control cells, \# $(P<0.001)$ compared to 2.2 and $4.5 \mathrm{mM}$ of VPA.

Fig. 3. Effect of different concentrations of VPA on the expression of MMP-2 and MMP-9 gene after $72 \mathrm{~h}$ incubation. $*(P<0.01), * *(P<0.001)$ compared to control cells, \# $(P<0.001)$ compared to 2.2 and $4.5 \mathrm{mM}$ of VPA. 
Fig. 4. Effect of different concentrations of VPA on the expression of Nm23-H1 and CD44v6 gene after 72 h incubation. * $(P<0.01), * *(P<0.001)$, compared to control cells, \# $(P<0.001)$ compared to 2.2 and $4.5 \mathrm{mM}$ of VPA

Fig. 5. Effect of VPA on the expression of Nm23-H1 and CD44v6 protein in A549 cell line. VPA increased and reduced the expression of $\mathrm{Nm} 23 \mathrm{H} 1$ and CD44v6 protein in A549 after $72 \mathrm{~h}$, respectively. 